\title{
PANDEMIC IMPACTS ON THE INTERNATIONAL ECONOMY
}

\section{GIORGI BENASHVILI}

\section{PhD student}

Ivane Javakhishvili Tbilisi State University, Georgia

giorgi.benashvili@tsu.ge

Abstract. In the history of world development, side to formation of the economic and financial systems, temporary crashes are not new. At different times, crises have always been experienced in different countries. However, within the rise of globalization and economic interdependence, modern crises are gaining much huge effects on social and economic systems.

More than half a year passed since the novel Coronavirus declared as a pandemic and virus already killed more than 900 000 people, while bringing the total number of infections to more than 28 million. Aside from widen spread of the virus, it can already be boldly said that virus has become the biggest challenge of modern reality since the World War II.

The Coronomic crisis, which became actual shortly since the outbreak of COVID-19 virus pandemic, is characterized by a different anatomy due to its nature. Unlike the previous crises, which experienced well-established financial and economic basis, the current crisis is significantly different. This time, the cause the crisis havenst financial of economic source, but the roots come from medicine. The current situation makes the economy a hostage of medicine (Papava, 2020) which is a dramatically different phenomenon from the previous crashes.

Unlike the economic crises over the last decade and century, the Coronomic crisis affects many sectors of the economy. While it puts into the agenda necessity of rethinking existing policy on international trade, production chain and generally structure of the economy. This requires in depth research, which is the aim of this article.

Without vaccine and effective clinical treatments agains the virus, social distancing remains the only recommended tool agains the virus. The latter is a significant challenge for the modern economy. The current situation, in addition to the necessity of rethink economic policy also puts into the agenda the issue of establishing new trends in the international trade and economy. The reality in the post-pandemic period, which may lead to changes in the international supply chain and a new dealing out of the supply market, will lead to significant changes in the international economy that requires in-depth research and analysis. The aim of the paper is to analyze some of the above issues, which will be presented on the based of existing empirical data.

\section{KEYWORDS: CORONAMIC; INTERNATIONAL ECONOMICS; INTERNATIONAL TRADE.}

For citation: Benashvili, G. (2020). Pandemic Impacts on the International Economy. Globalization and Business, 10. $192-197$. (In Georgian). https://doi.org/10.35945/gb.2020.10.024 


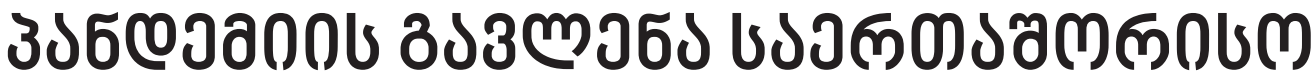 วзмбща0з১80}

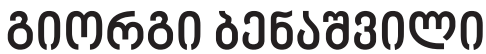

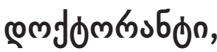

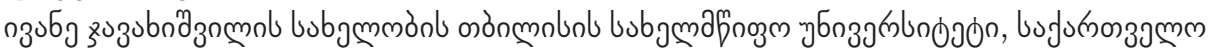

giorgi.benashvili@tsu.ge

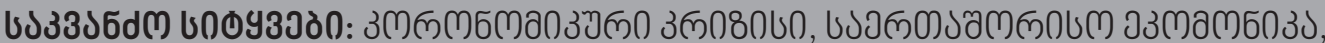

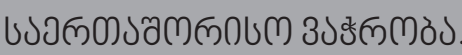

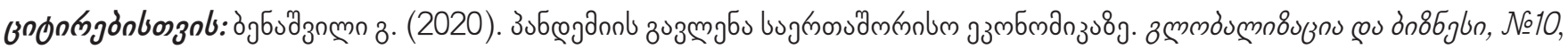
33. 192-197. https://doi.org/10.35945/gb.2020.10.024

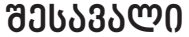

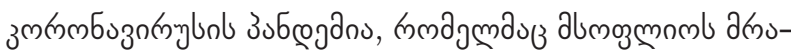
зum Јзау

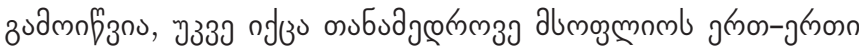

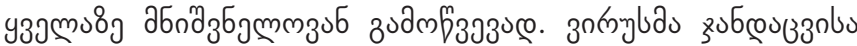

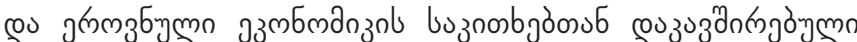

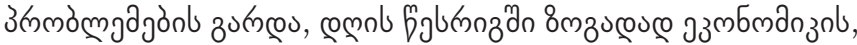

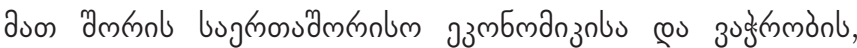
buznorbjóng gusygbu.

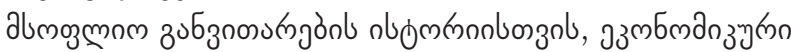

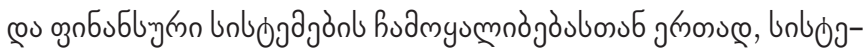

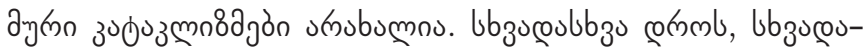
ubzu J3gys

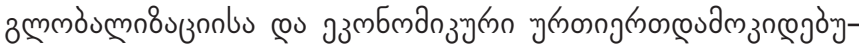

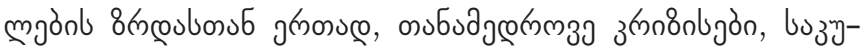

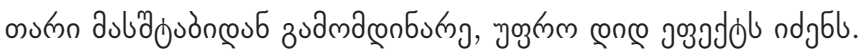

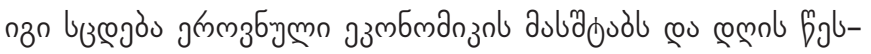

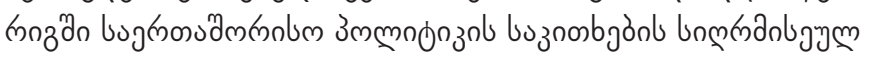
zubbnmzal agjбabl.

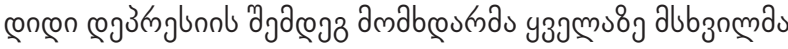
оुnбubling

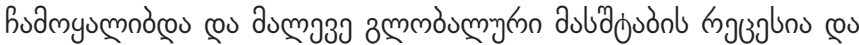

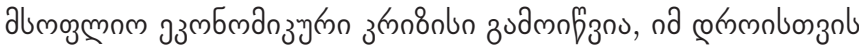

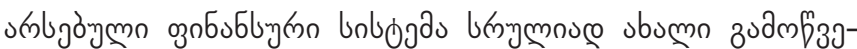

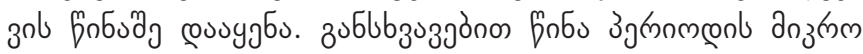

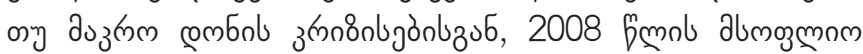

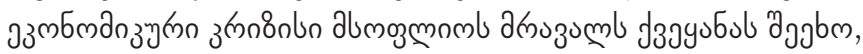

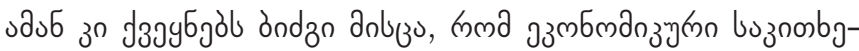

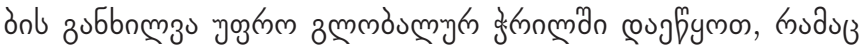

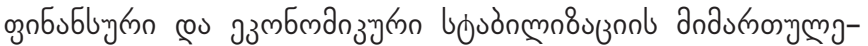

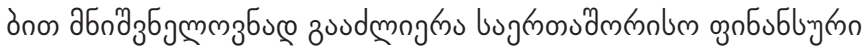

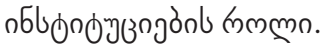

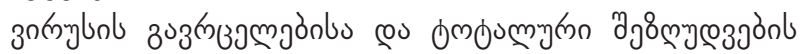

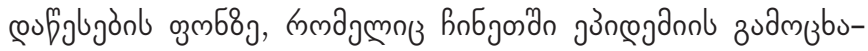

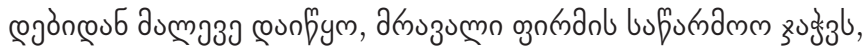

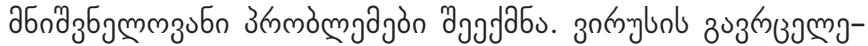

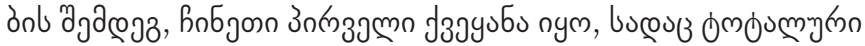

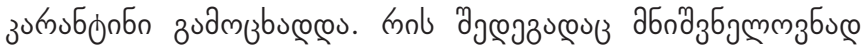

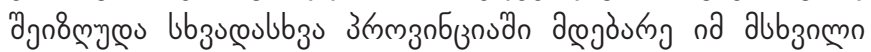

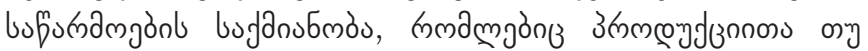

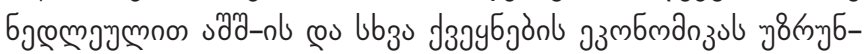

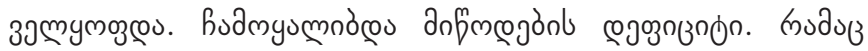

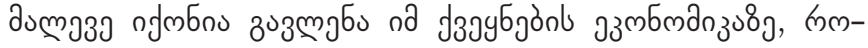

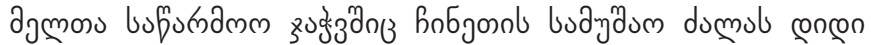

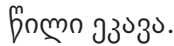

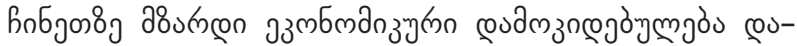

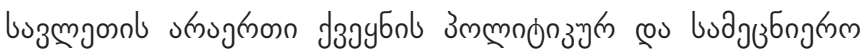

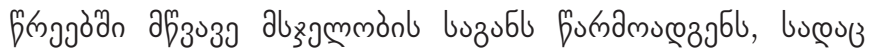

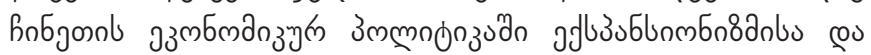

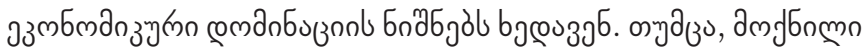

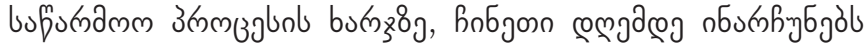

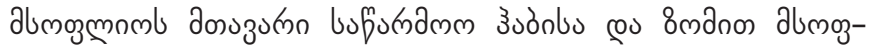

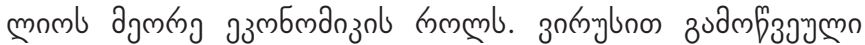

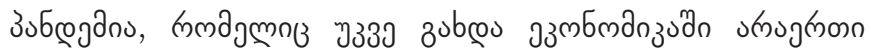

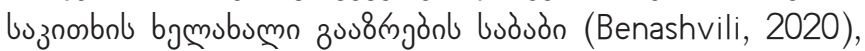

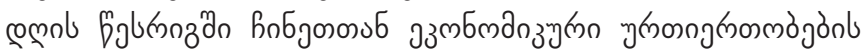

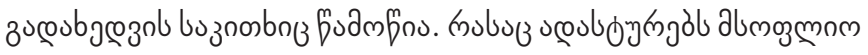

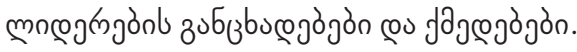

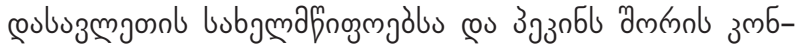

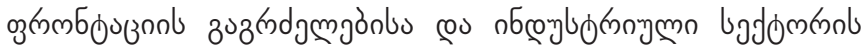

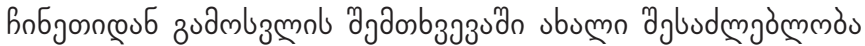




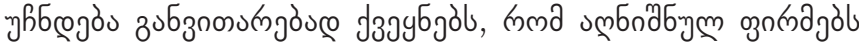

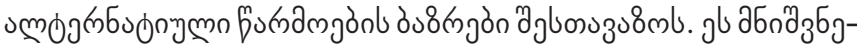

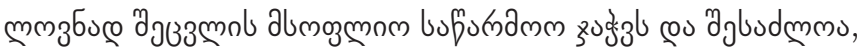

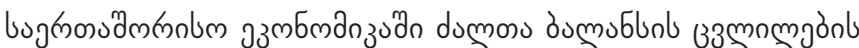
buogydzjmanz zubegl.

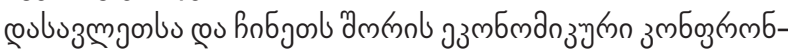

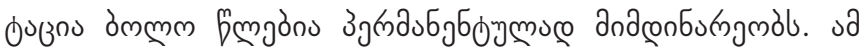

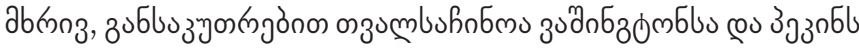

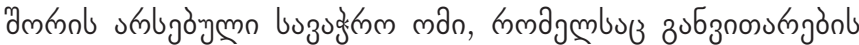

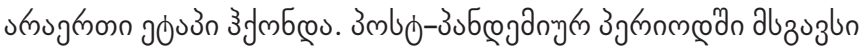

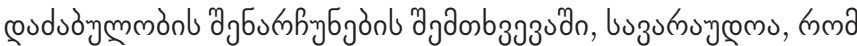

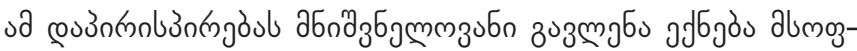
мпм эзмбмдпзово

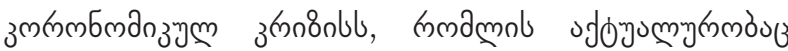

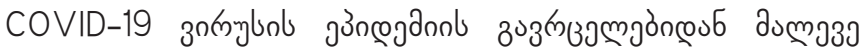

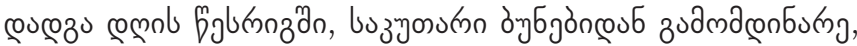

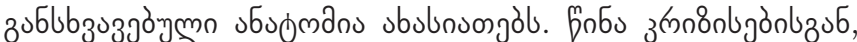

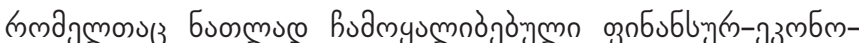

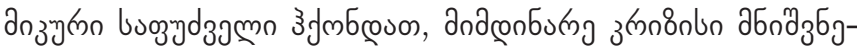

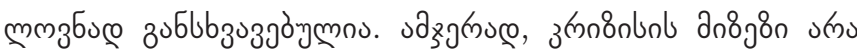

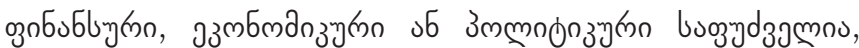

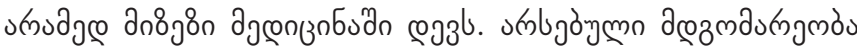

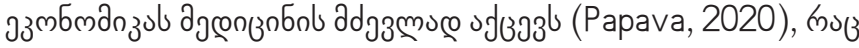

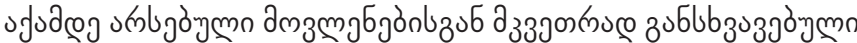

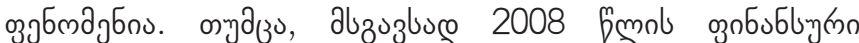

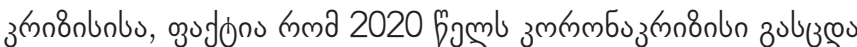

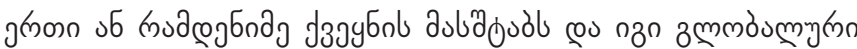

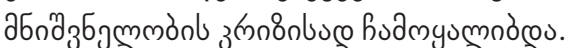

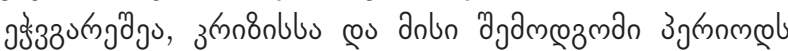

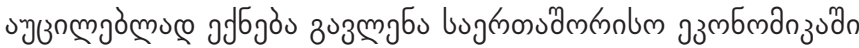

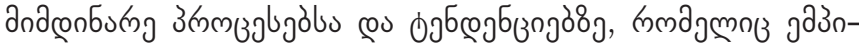

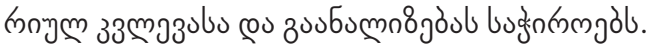

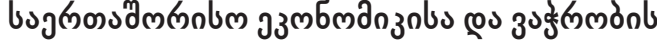

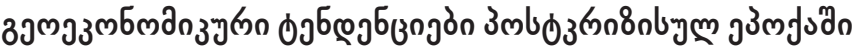

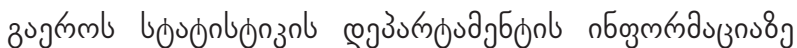

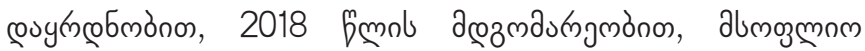

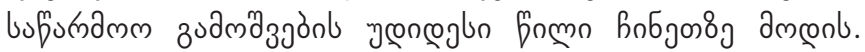

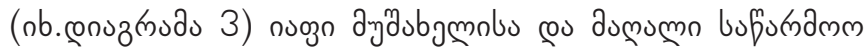

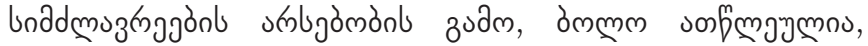

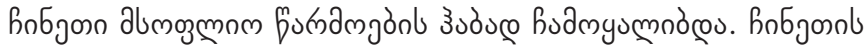

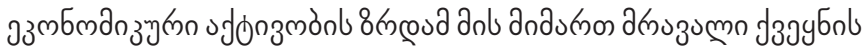

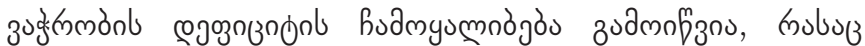

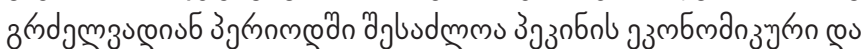

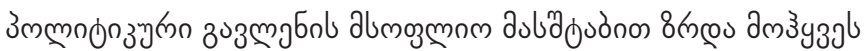
(Benashvili, 2020).

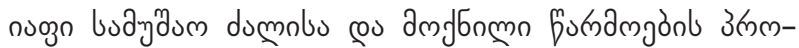

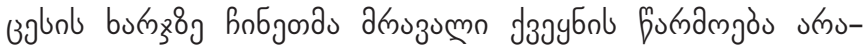

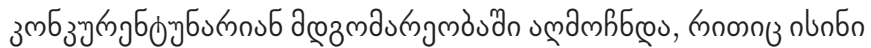

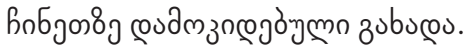

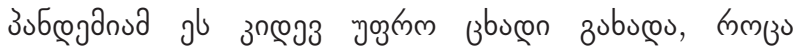

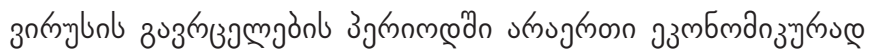

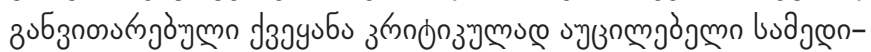

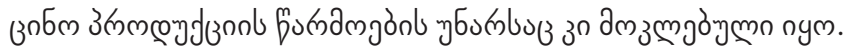

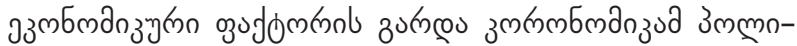

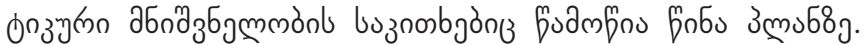

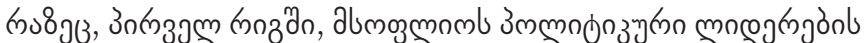

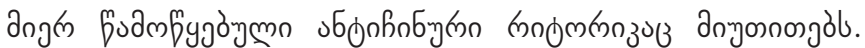

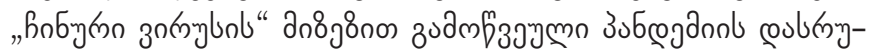

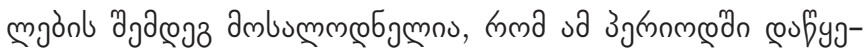

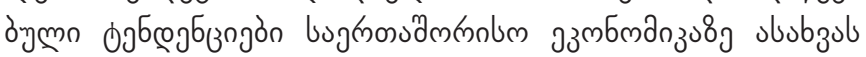

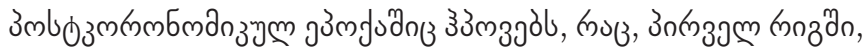

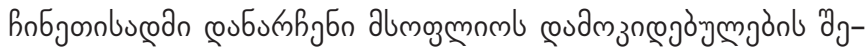
उзмs dmyзjòs.

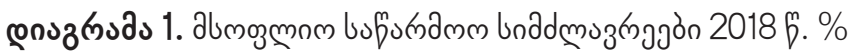

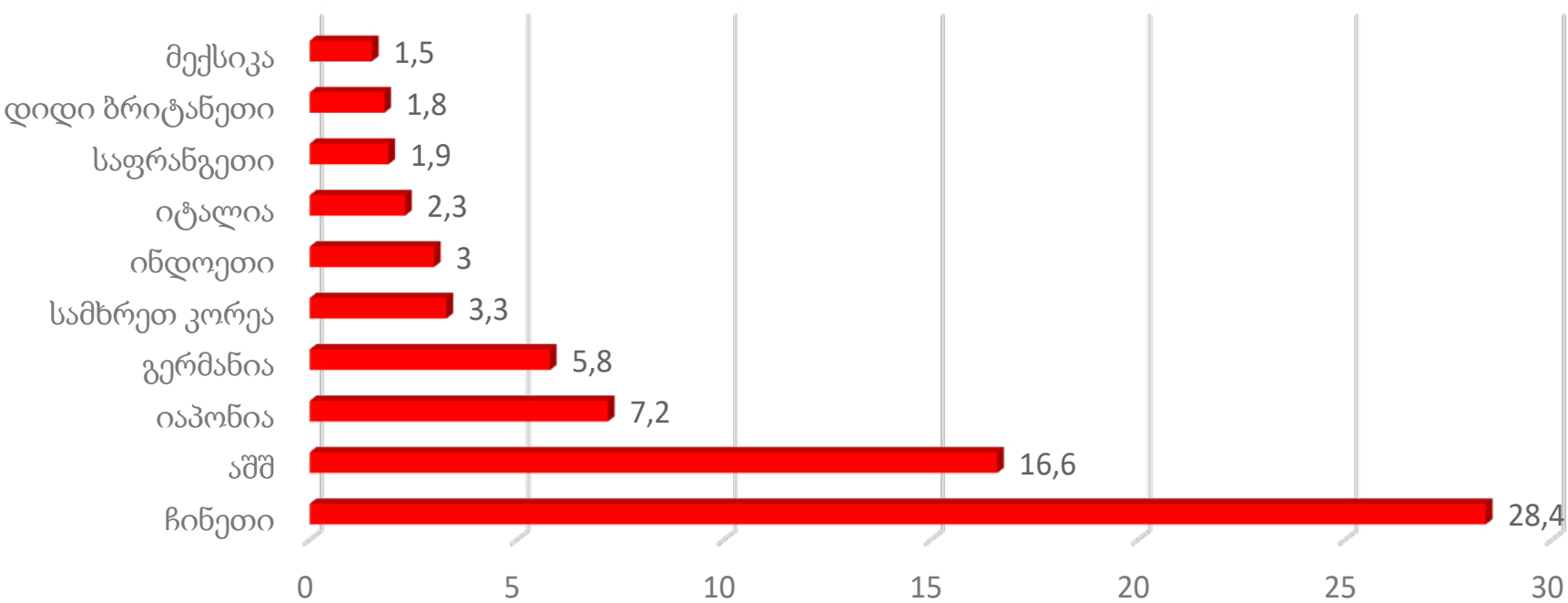

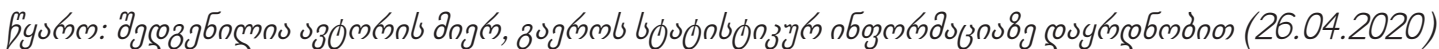




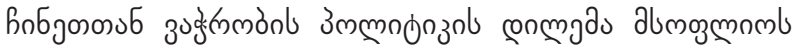

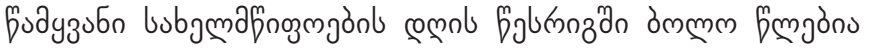

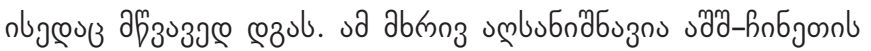

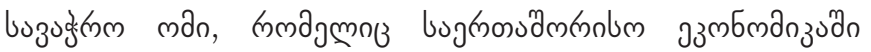

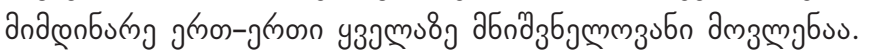

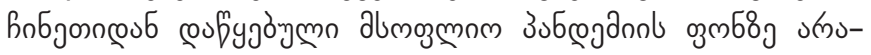

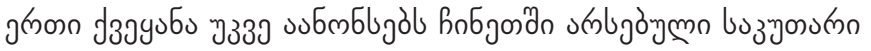

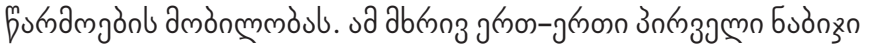

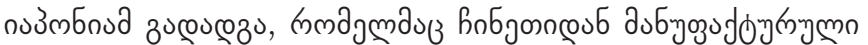

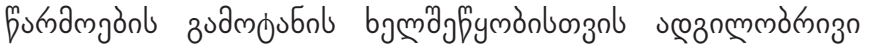

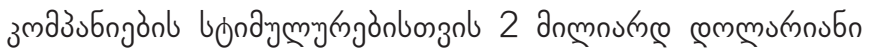

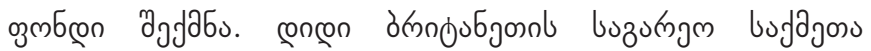

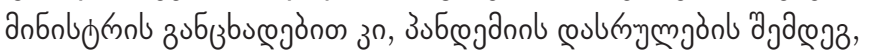

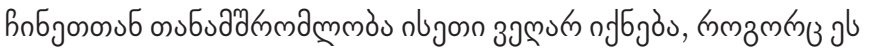

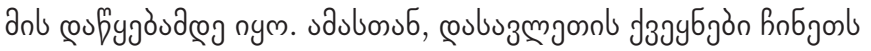

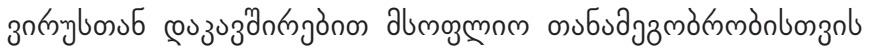

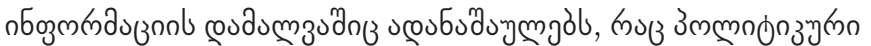

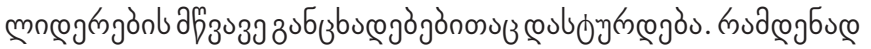

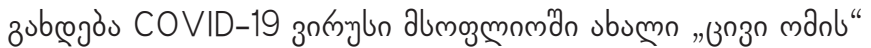
œufy

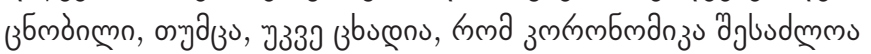

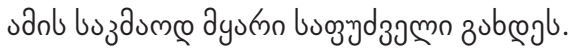

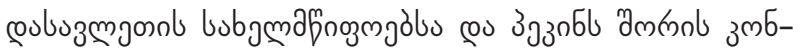

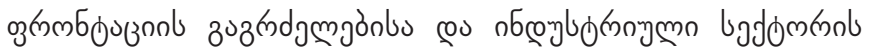

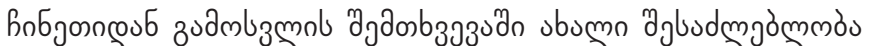

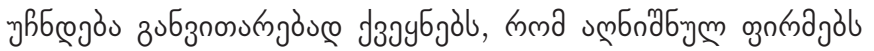
uмmog

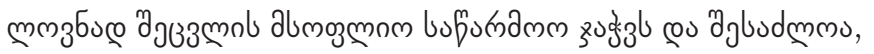

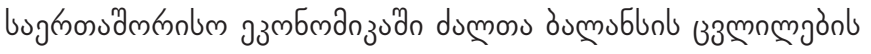
buogydzjmang zubegl.

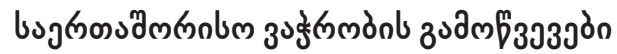

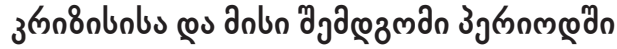

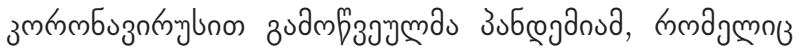

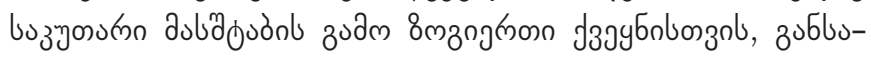

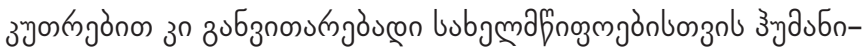

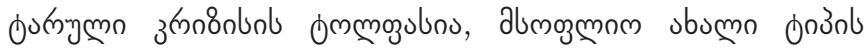

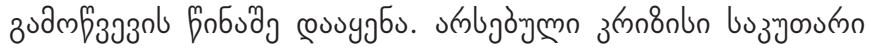

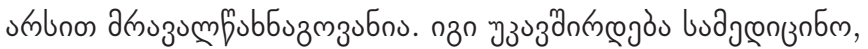

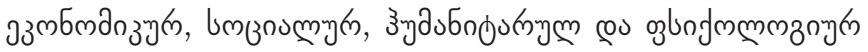
buznorbjoll.

з мmलठ̀

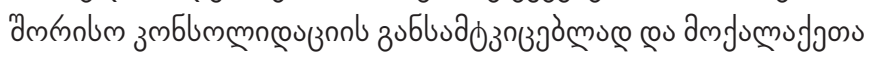

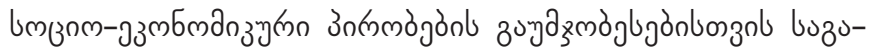

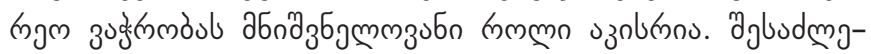

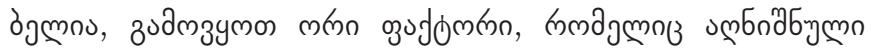

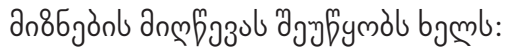

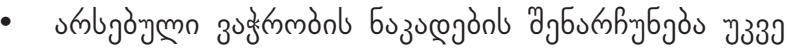

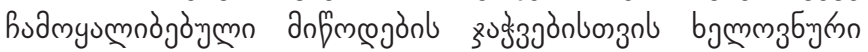

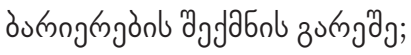

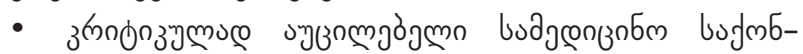

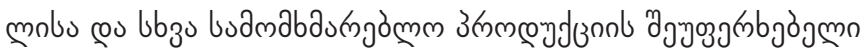

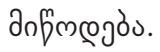

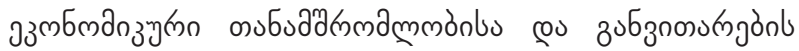

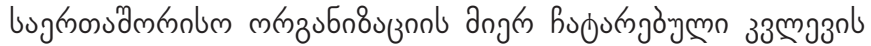

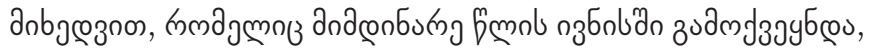

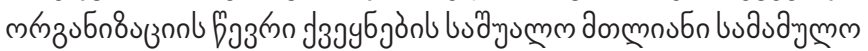

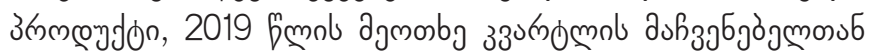

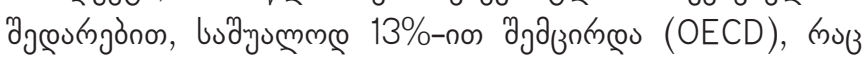

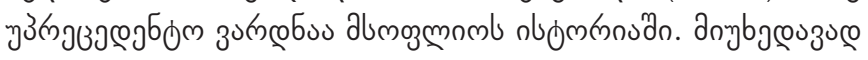

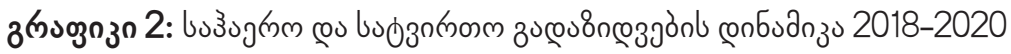

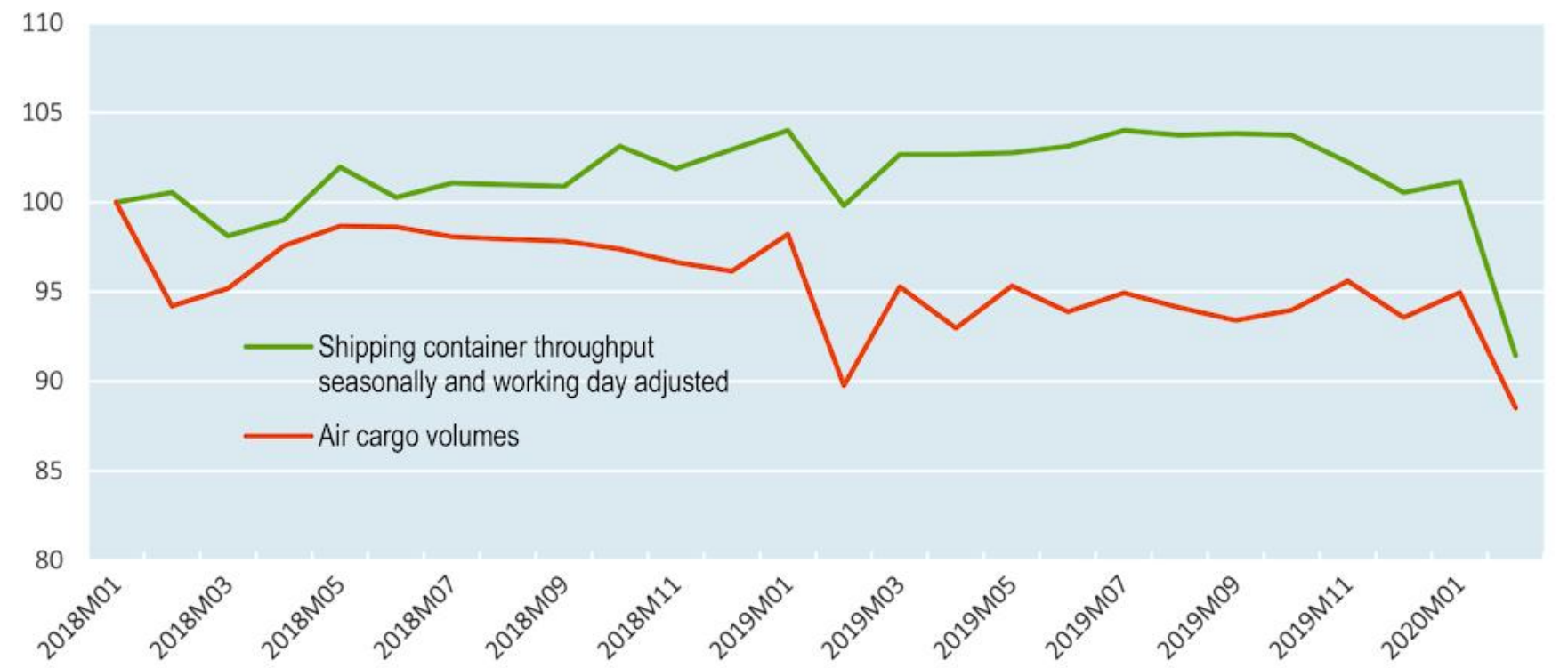

fyornm: oecd.org 


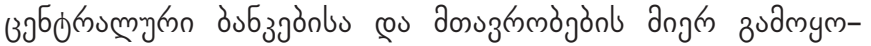

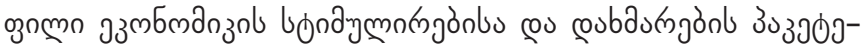

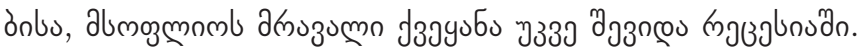

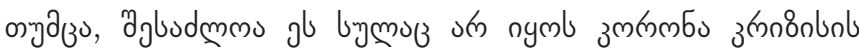
y3amoso yumgln b

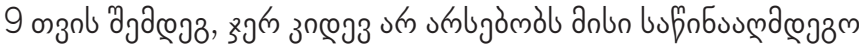

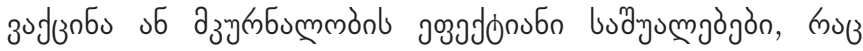

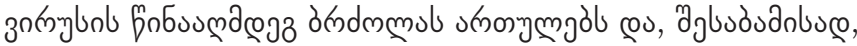

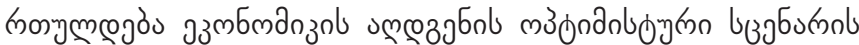
зubbminzogmgosos.

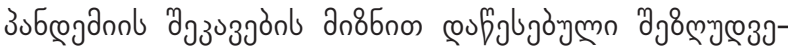

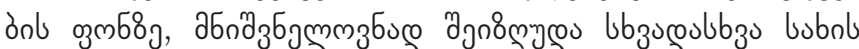

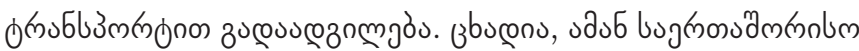

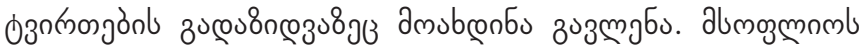

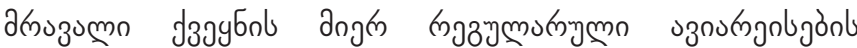

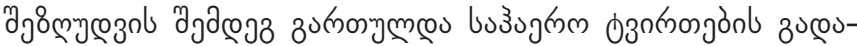

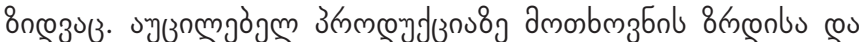

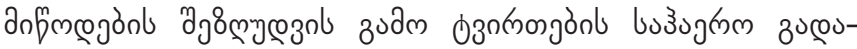

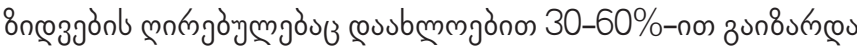
cos zun8um nos anfmmegònl comm(3. (Curran, 2020)

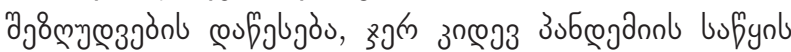

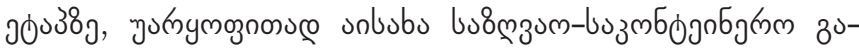
एо

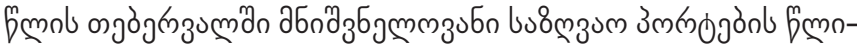

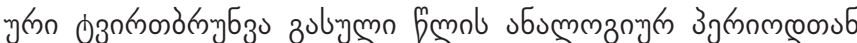

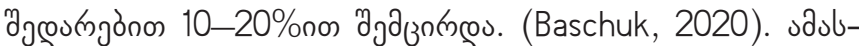

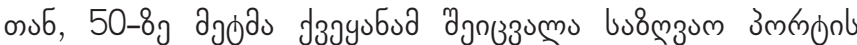

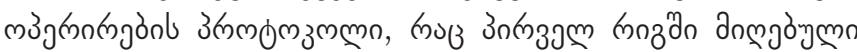

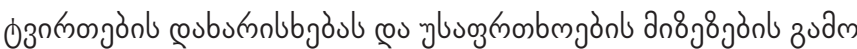

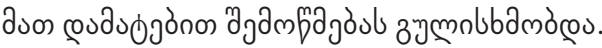

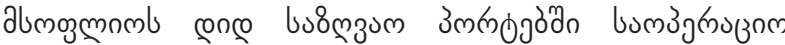

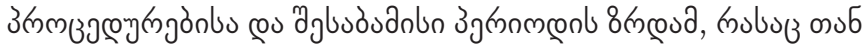

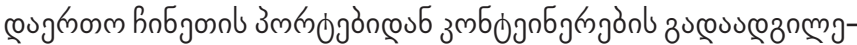

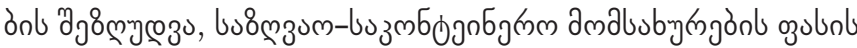

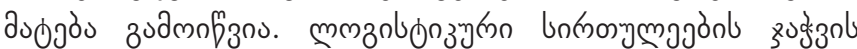

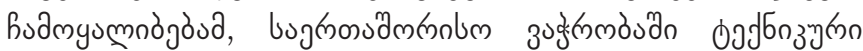

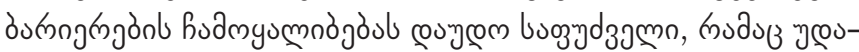

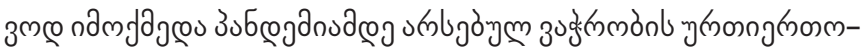

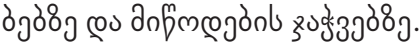

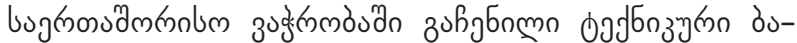

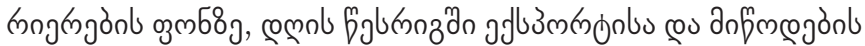

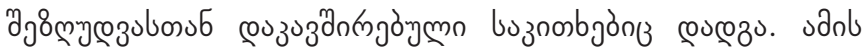

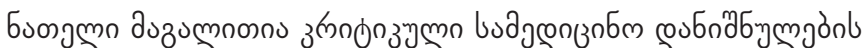

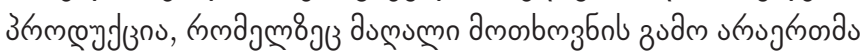

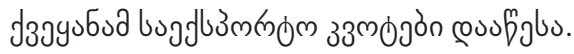

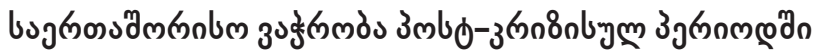

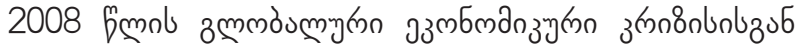

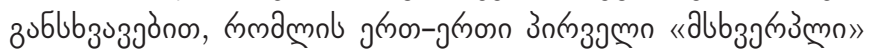

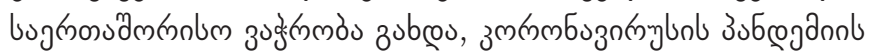

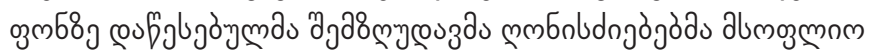

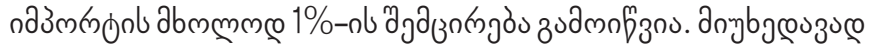

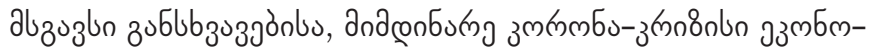

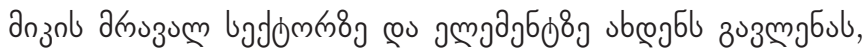

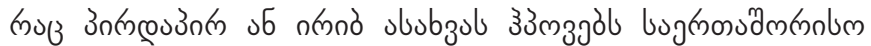

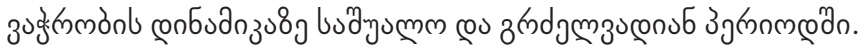

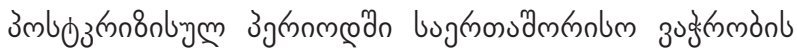

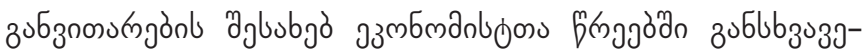

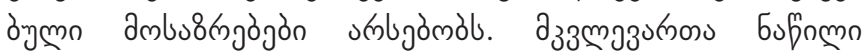

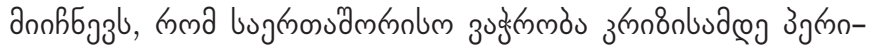

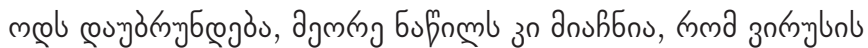

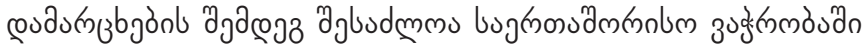

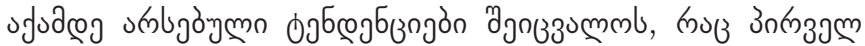

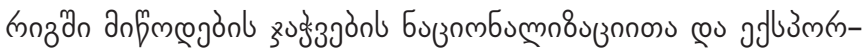

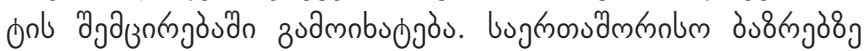
ง

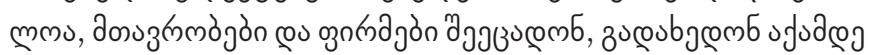

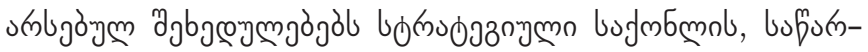

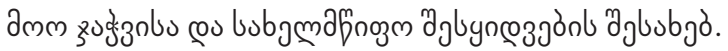

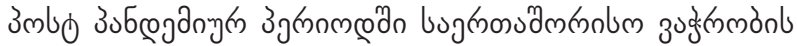

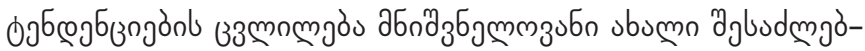

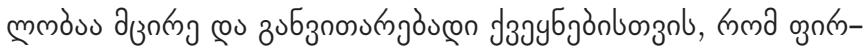

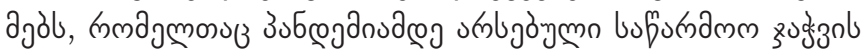

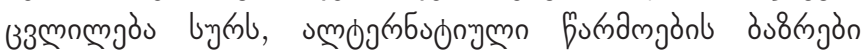

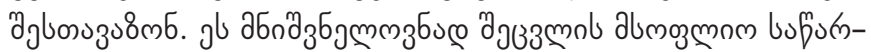

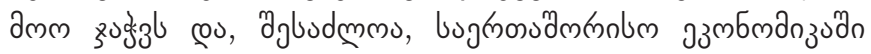

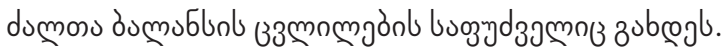

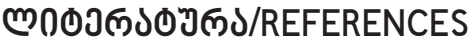

Alwis, Ajith (2020). Coronomics - plan your eggs and basket, Daily FT, http://www.ft.lk/columns/Coronomics--Plan-your-eggs-and-the-basket-/4-695109 (last seen 05.06.2020)

Baschuk, B. (2020), "A Trade Collapse that's Heading Into the History Books", Bloomberg, https://www.bloomberg. $\mathrm{com} /$ news/articles/2020-03-26/supply-chain-latest-a-trade-plunge-worthy-of-the-history-books (Last seen 08.09.2020)

Benashvili, G. (2020). China's one belt and road initiative its prospects for Georgia, Ecoforum, Vol. 9, Issue 1(21) http://www.ecoforumjournal.ro/index.php/eco/article/view/1043 (last seen 28.04.2020) 
Makris, Miltos (2020) Coronomics: how a pandemic can impact world economics University of Kent News Centre, https://www.kent.ac.uk/news/society/24809/coronomics-how-a-pandemic-can-impact-world-economics last seen 28.04.2020)

Calder, S. (2020) Airlines preparing for future with fewer passengers and higher fares, Independent.co.uk https://www.independent.co.uk/travel/news-and-advice/coronavirus-flight-prices-airline-passengersfares-a9464821.html (last seen 19.04.2020)

Curran, E. (2020), "Urgent Demand for Medical Equipment is Making Air Cargo Fees "Absolutely Crazy"”, Bloomberg, https://www.bloomberg.com/news/articles/2020-03-30/-absolutely-crazy-air-cargo-fees-highlight-supplychain-squeeze (Last seen 09.09.2020)

Evenett, S. (2020), "Tackling COVID-19 Together", Global Trade Alert, University of St. Gallen, Switzerland, https:// www.globaltradealert.org/reports (Last seen 10.09.2020)

OECD (2020), COVID-19 and international trade: Issues and actions http://www.oecd.org/coronavirus/policyresponses/covid-19-and-international-trade-issues-and-actions-494da2fa/ (Last seen 10.09.2020)

Reynolds, I. (2020) Japan to fund firms to shift production out of China, Bloomberg https://www.bloomberg.com/ news/articles/2020-04-08/japan-to-fund-firms-to-shift-production-out-of-china (last seen 24.04.2020)

Mello-Théry, N. \& Théry, H. (2020). The geopolitics of COVID--19. Espaço e Economia. https://journals.openedition. org/espacoeconomia/11224 (27.04.2020)

Papava, V. \& Charaia, V. (2020). The Coronomic Crisis and Some Challenges for the Georgian Economy. Rondeli Foundation https://www.gfsis.org/publications/view-opinion-paper/136 (last seen 24.04.2020)

Papava, V.. (2020). Coronomic Crisis: When The Economy Is A Hostage To Medicine. eurasiareview, https://www. eurasiareview.com/29032020-coronomic-crisis-when-the-economy-is-a-hostage-to-medicine-oped/(last seen 25.04.2020)

Papava, V. \& Silagadze, A. (2019). "On the Georgian name of one key economic term "Gross Domestic Product", 203204.(in Georgian) Journal Globalization and Business https://www.researchgate.net/publication/334897039_ rogor_unda_itargmnos_kartulad_termini_GROSS_DOMESTIC_PRODUCT (25.04.2020)

Creitz, Ch. (2020) Pompeo blames China, Fox News https://www.foxnews.com/media/pompeo-china-coronaviruscoverup-loss-of-life (last seen 25.04.2020)

Georgian Tourism Statistical Review 2018 https://gnta.ge/ge/publication/2018-geo/ (last seen 20.04.2020)

Rodrik, D. (2020) Will COVID-19 Remake the World? Project Syndicate https://www.project-syndicate.org/ commentary/will-covid19-remake-the-world-by-dani-rodrik-2020-04 (27.04.2020)

El-Erian, M. (2020) Saving the developing world from COVID-19 Project Syndicate https://www.projectsyndicate.org/commentary/covid19-aid-to-developing-countries-by-mohamed-a-el-erian-2020-04 (last seen 28.04.2020)

Economic Impact Reports, World Travel \& Tourism council wttc.org/Research/Economic-Impact (last seen 24.04.2020)

World Bank Database https://data.worldbank.org/indicator/SH.XPD.CHEX.GD.ZS (last seen 26.04.2020) 